\title{
Initial Solidification Behavior of Ultra Low, Low and Middle Carbon Steel
}

\author{
Hideo MIZUKAMI, Sei HIRAKI, Masayuki KAWAMOTO and Tadao WATANABE
}

Corporate Research \& Development Labs., Sumitomo Metal Industries, Ltd., Hasakimachi, Kashima-gun, Ibaraki-ken, $314-0255$ Japan.

(Received on June 1, 1999; accepted in final form on August 20,1999)

\begin{abstract}
The cooling curves at initial stage of solidification of carbon steel sample were measured using a new temperature measurement system that consisted of a two-dimensional optical pyrometer. Undercooling and recalescence phenomena were observed on the measured cooling curve of ultra low, low and middle carbon steel samples, and the solidification sequence at initial stage of solidification was shown to be different from normal solidification. A numerical analysis employing the dendrite tip growth model has been constructed, and fitted to a measured cooling curve by the parameter of interfacial heat transfer coefficient, and then the interfacial heat transfer coefficient between sample and chill plate which made of a transparent sapphire glass can be predicted accurately. The evenness of solidified shell is made clear to be influenced mainly by thermal deformation.
\end{abstract}

KEY WORDS: carbon steel; optical measurement; undercooling; recalescence; deformation; nucleation; dendrite growth; heat transfer.

\section{Introduction}

Understanding of the initial stage of solidification for ultra low carbon steel is necessary to stabilize and improve surface quality of continuously cast slabs. For the solidification mechanism of low carbon and middle carbon steel, many studies $^{1-5)}$ have been carried out to investigate experimentally and analytically, and it has become clear that the unevenness of solidified shell growth was caused by $\delta / \gamma$ transformation during solidification. On the other hand, solidification of ultra low carbon steel has only just been studied, the mechanism of shell growth at initial stage of solidification has been made somewhat clearer. However, initial solidification of carbon steel occurs within very short time near the meniscus in the mold, it seemed to be hard to estimate accurately the behavior during solidification by the previous experimental technique using a thermocouple.

In the present paper, a new experimental technique which use with a two-dimensional optical pyrometer is presented for measurement of the variation of surface temperature of ultra low carbon steel sample with time. The behavior of the surface temperature of ultra low carbon steel is compared with that of low carbon and middle carbon steel samples, and the characteristics of solidification phenomena of ultra low carbon steel was discussed. Furthermore, a numerical model for the initial stage of solidification is described which employs the dendrite tip growth model, from which the interfacial heat transfer coefficient between the sample and the chill plate which made of a transparent sapphire glass can be predicted. From both the experimental and numerical results, the behavior of cooling curve at the sample surface/chill plate with time during and after solidification is investigated, and the phenomena of initial stage of solidification is discussed.

\section{Experimental Procedures}

\subsection{Composition of Samples}

The chemical compositions of the carbon steel samples are listed in Table 1. The $150 \mathrm{~kg}$ steel ingots were produced by electrolytic iron and alloying elements in a vacuum furnace, and then were hot forged. Samples were machined into round bars of $50 \mathrm{~mm}$ in diameter and $150 \mathrm{~mm}$ in length from the ingots. In order to investigate the effect of carbon content on cooling conditions at the initial stage of solidification, carbon content was varied between 0.0005 and 0.14 mass \%. Sample ULCl and ULC2 are ultra low carbon steel which are different carbon contents. Sample LC is low carbon steel and sample $\mathrm{MC}$ is middle carbon steel.

\subsection{Experimental Apparatus}

Figure 1 shows a schematic diagram of experimental

Table 1. Chemical composition of samples. (mass\%)

\begin{tabular}{l|r|r|r|r|r}
\hline \hline Sample & \multicolumn{1}{|l|}{ C } & \multicolumn{1}{l|}{ Si } & \multicolumn{1}{l|}{ Mn } & P & \multicolumn{1}{l}{} \\
\hline ULC1 & 0.0005 & 0.01 & 0.12 & 0.014 & 0.003 \\
\hline ULC2 & 0.002 & 0.01 & 0.11 & 0.012 & 0.003 \\
\hline LC & 0.04 & 0.04 & 0.19 & 0.026 & 0.006 \\
\hline MC & 0.14 & 0.16 & 0.54 & 0.016 & 0.003 \\
\hline
\end{tabular}




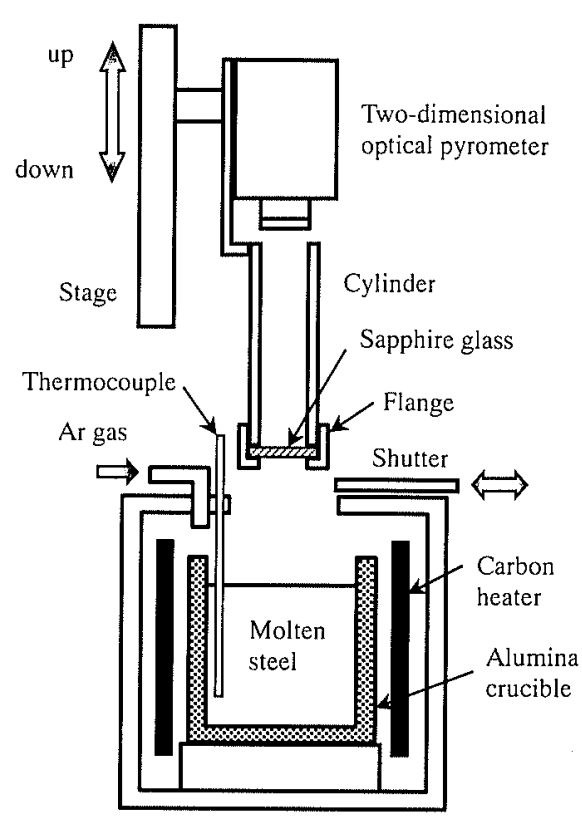

Fig. 1. Schematic view of the temperature measurement system.

apparatus which measure the cooling curve of the sample surface during the initial stage of solidification. The experimental apparatus consists of a two-dimensional optical pyrometer, a chill plate which made of transparent sapphire glass, a cylinder, a movement stage for up and down and a tammann furnace. A lens of an optical pyrometer was made of germanium and a central transmission wave length of the lens was in the range of 3 to $5 \mu \mathrm{m}$. In order to match with the central transmission wave length of the lens, a sapphire glass was chosen as a chill plate. The chill plate was fixed at the end of the cylinder by a flange and then, this cylinder was fixed to an optical pyrometer, therefore the distance between chill plate and optical pyrometer was always constant and so the optical pyrometer was alwaysfocussed for measuring temperature during experiments. The approximately $2 \mathrm{~kg}$ sample was set in an aluminum crucible and melted in a tammann furnace under an argon gas atmosphere with the shutter on the furnace closed. The temperature of molten steel was controlled by an $\mathrm{R}$ type thermocouple and adjusted to liquidus temperature $+20 \mathrm{~K}$. After opening the shutter, the chill plate was moved down and contacted the molten steel for $2 \mathrm{~s}$, and then the chill plate was moved up. Each successive operation was carried out automatically by a programmable controller. While the chill plate touched the molten steel, the temperature of the sample surface was measured with a two-dimensional optical pyrometer through the transparent sapphire glass. Output voltage of the photocell in the optical pyrometer was recorded directly by a digital memory, and then the output voltage was converted to temperature on a computer interfaced to the digital memory after the experiment. In this way, the response time of the measurement system for temperature was made less than $20 \mathrm{~ms}$.

A solidified shell was obtained after moving up the chill plate. An inner surface of a flange to fix the chill
Table 2. Experimental conditions.

\begin{tabular}{l|l|l}
\hline \hline \multirow{2}{*}{ olten steel } & Teight & 2Kg \\
\cline { 2 - 3 } & Temperature & Liquidust20K \\
\hline Atmosphere & Ar gas & \multicolumn{2}{|l}{} \\
\hline Dipping time & 2s & Sapphire glass \\
\hline Chill plate & Material & $\phi 30 \times 5 \mathrm{~mm}^{\mathrm{T}}$ \\
\cline { 2 - 3 } & Size & $\mathrm{R}_{\text {nax }}<0.9 \mu \mathrm{m}$ \\
\cline { 2 - 3 } & Roughness of surface \\
\hline \multirow{2}{*}{$\begin{array}{l}\text { Two-dimensional } \\
\text { optical pyrometer }\end{array}$} & Sampling time & $20 \mathrm{~ms}$ \\
\cline { 2 - 3 } & Resolution & $0.1 \mathrm{~mm}$ \\
\cline { 2 - 3 } & Accuracy & $\pm 1 \mathrm{~K}$ \\
\hline
\end{tabular}

plate was coated with alumina cement except for about $5 \times 10^{-3} \mathrm{~m}$ in length. A solidified shell could not be attached to the coated region with alumina cement in the flange and was prevented to constrained by the flange because of shrinkage during solidification. The noncoated region in the flange was polished with emery paper to roughen, and was attached by a solidified shell. In this way, it made possible to obtain a solidified shell without a constrained force.

Experimental conditions and specifications of measurement system are listed in Table 2 . The surface of the chill plate was made flat to less than $0.9 \mu \mathrm{m}$ to avoid the effect of heterogeneous nucleation at projections on the chill plate. In this experiment, it was feared that the emission of the transparent sapphire glass in the infrared wavelength band changed with time, because the chill plate touched with molten steel directly. In order to estimate the measuring error which was caused by the change of emissivity, the surface temperature of molten steel in the crucible was measured with a used transparent sapphire glass, but the difference between the temperature of molten steel measured with a thermocouple and the temperature measured with the optical pyrometer was less than $2 \mathrm{~K}$. It was cleared that the measurement system had good accuracy. In each experiment, a new sapphire glass was used.

\section{Numerical Analysis for Initial Stage of Solidification after Nucleation}

A solidification analysis was divided into two cases that were accompanied with undercooling or not.

When a region of undercooling near the sample surface disappeared and a temperature gradient at the solid/liquid interface became positive, the temperature recovery method was adopted for the numerical analysis of solidification.

When a region of undercooling existed near the sample surface, the relationship between a growth velocity and a dendrite tip temperature was predicted using the dendrite growth model ${ }^{6-9)}$ and then this relationship was substituted in the numerical analysis. Specifically, a temperature at the solid/liquid interface was calculated from a heat flow equation and a growth velocity of dendrite tip was calculated by the dendrite growth model.

The dendrite growth model has been constructed under binary alloy system. An influence of alloying elements on a primary dendrite arm spacing and a solidified morphology in low carbon steel was cleared by Esaka et 
al. ${ }^{10)}$ But an influence in ultra low carbon and middle carbon steel has not been cleared. In this way, it was simply analyzed using the dendrite growth model under $\mathrm{Fe}-\mathrm{C}$ binary system.

In the dendrite growth model, the total undercooling is expressed as

$$
\Delta T=\Delta T_{\mathrm{c}}+\Delta T_{\mathrm{r}}+\Delta T_{\mathrm{k}}+\Delta T_{\mathrm{t}}
$$

where $\Delta T$ is the total undercooling, $\Delta T_{\mathrm{c}}$ is the solute undercooling, $\Delta T_{\mathrm{r}}$ is the curvature undercooling, $\Delta T_{\mathrm{k}}$ is the kinetic undercooling, and $\Delta T_{\mathrm{t}}$ is the thermal undercooling.

The dendrite tip radius is determined by marginally stability criterion

$$
r=\frac{4 \pi^{2} \Gamma}{-\frac{2 m_{\mathrm{v}} C_{0} P_{\mathrm{c}}(1-k) \xi_{\mathrm{c}}}{1-\left(1-k_{\mathrm{v}}\right) I_{\mathrm{v}}\left(P_{\mathrm{c}}\right)}+\frac{\Delta H \xi_{1}}{C_{\mathrm{p}}}}
$$

where $r$ is the dendrite tip radius, $\Gamma$ is the Gibbs-Thomson coefficient, $C_{0}$ is the initial concentration, $m_{\mathrm{v}}$ is the liquidus slope at high growth rate, $\Delta H$ is the heat of fusion, $C_{\mathrm{p}}$ is the specific heat, $I_{\mathrm{v}}$ is the Ivantsov function, $P_{\mathrm{c}}$ is the solute Peclet number, $k$ is the equilibrium distribution coefficient, $k_{\mathrm{v}}$ is the non-equilibrium distribution coefficient, ${ }^{11)} \xi_{\mathrm{c}}$ and $\xi_{\mathrm{t}}$ is the stability terms. ${ }^{12)}$

The dendrite tip temperature and the dendrite tip concentration are determined by

$$
\begin{gathered}
T_{\mathrm{tip}}=T_{\mathrm{L}}+m_{\mathrm{v}} C_{\mathrm{L}}^{*}-\frac{r T_{\mathrm{L}}^{2} V}{\Delta H V_{0}}-\frac{2 \Gamma}{r} \\
C_{\mathrm{L}}^{*}=\frac{C_{0}}{1-\left(1-k_{\mathrm{v}}\right) I_{\mathrm{v}}\left(P_{\mathrm{c}}\right)}
\end{gathered}
$$

where $T_{\text {tip }}$ is the dendrite tip temperature, $V$ is the growth velocity, $T_{\mathrm{L}}$ is the liquidus temperature, $V_{0}$ is the limiting crystallization velocity and $C_{\mathrm{L}}^{*}$ is the concentration at dendrite tip.

Figure 2 shows the relationship between growth velocity of dendrite tip and undercooling of dendrite tip for sample ULC1, ULC2, LC and MC. The difference of growth velocity for each sample was a little under the calculated conditions in this study. The tip velocity increased abruptly near zero undercooling, and the tip velocity went from zero to about $1 \times 10^{2} \mathrm{~ms}^{-1}$ as undercooling went from zero to about $50 \mathrm{~K}$ for each sample. The curves for sample ULC1 and ULC2 were smooth curves. The curves for sample LC and $\mathrm{MC}$ showed slightly buckling near $5 \mathrm{~K}$ and $20 \mathrm{~K}$, respectively. Above this temperature, both curves matched those for sample ULC1 and ULC2.

The numerical technique adopted in this study was the variable space network method from Murray et al. ${ }^{13)}$ The major feature of this method is that the interface position between solid and liquid is always known.

As the length of primary solidified grid must be a finite value, the length was set at $1 \times 10^{-6} \mathrm{~m}$ which was smaller than primary dendrite arm spacing. Furthermore, as cooling conditions were controlled by heat transfer coefficient between the chill plate and the sample surface, the temperature of the chill plate was constant at $303 \mathrm{~K}$.

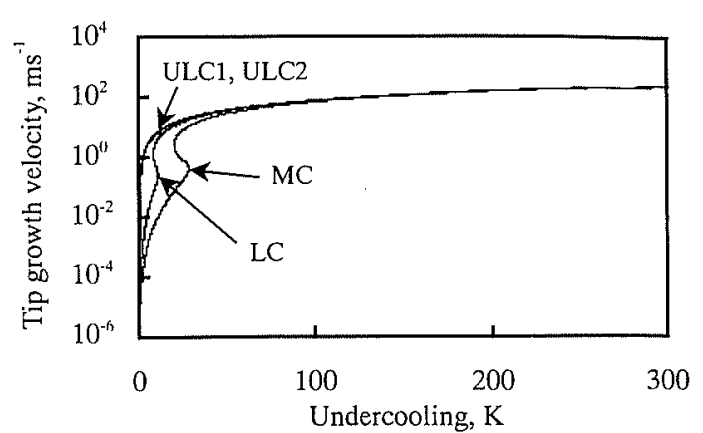

Fig. 2. Change of dendrite tip growth velocity with undercooling of carbon steel.

The heat flow equation in both solid and liquid region are written in differential form by, respectively

$$
\begin{aligned}
& \frac{T_{i}^{t+\Delta t}-T_{i}^{t}}{\Delta t}= \frac{i}{2 R}\left(T_{i+1}-T_{i-1}\right) V \\
&+k_{\mathrm{S}}\left(\frac{N}{R}\right)^{2}\left(T_{i+1}-2 T_{i}+T_{i-1}\right)+\frac{V \Delta t \Delta H}{\rho C_{\mathrm{p}} \Delta x} \ldots \ldots . . \\
& \frac{T_{i}^{t+\Delta t}-T_{i}^{t}}{\Delta t}= \frac{M-i}{2(L-R)}\left(T_{i+1}-T_{i-1}\right) V \\
&+k_{\mathrm{L}}\left(\frac{M-N}{L-R}\right)^{2}\left(T_{i+1}-2 T_{i}+T_{i-1}\right)
\end{aligned}
$$

where $T$ is the temperature, $R$ is the position of interface between solid and liquid, $L$ is the shell thickness after solidification, $\rho$ is the density, $\Delta x$ is the mesh size, $k_{\mathrm{S}}$ and $k_{\mathrm{L}}$ are the thermal diffusivity in solid and liquid respectively and in this calculation these thermal diffusivity were same value, $M$ and $N$ is the number of grids in each phase respectively and $i$ is the grid number. In this calculation, $L$ was $5 \times 10^{-3} \mathrm{~m}, M$ and $N$ were 250 meshes.

The sample was monotonously cooled from one side just before nucleation, and when the temperature of sample surface reached the nucleation temperature, the calculation for solidification in undercooled liquid was started. The nucleation temperature was determined to be the temperature for maximum undercooling on the cooling curve.

The analysis was carried out based on the assumption that primary dendrite arm spacing near the sample surface was sufficiently small in comparison with the size of analytical region, and the direction of dendrite growth was unidirectional and the isothermal line determined with dendrite tip temperature was parallel to the sample surface.

The time interval used in this numerical analysis has to be limited according to stability criteria. Before solidification starts after the nucleation event, the stability is defined ${ }^{14)}$ by

$$
\Delta t=\frac{\rho C_{\mathrm{p}}}{2 \alpha}\left(\frac{h}{2 \alpha \Delta x}+\frac{1}{\Delta x^{2}}\right)^{-1}
$$

where $\Delta t$ is the increment time, $\alpha$ is the thermal conductivity, $h$ is the heat transfer coefficient.

After the solidification process starts, the stability is 
Table 3. Physical properties used in calculations.

\begin{tabular}{|c|c|c|c|c|c|c|}
\hline Parameter & Unit & ULCI & ULC2 & LC & $\mathrm{MC}$ & Ref. \\
\hline $\mathrm{C}_{0}$ & mass \%C & 0.0005 & 0.002 & 0.04 & 0.14 & - \\
\hline$T_{L}$ & $\mathrm{~K}$ & 1808 & 1808 & 1807 & 1797 & \multirow[t]{4}{*}{ 15) } \\
\hline $\mathrm{m}$ & $\mathrm{Kmass}^{-1}$ & \multicolumn{4}{|l|}{-81.1} & \\
\hline $\mathrm{k}$ & - & \multicolumn{4}{|l|}{0.16} & \\
\hline$V_{i}$ & $\times 10^{6}, \mathrm{~m}^{3} \mathrm{~mol}^{-1}$ & 7.669 & 7.669 & 7.656 & 7.616 & \\
\hline$\triangle \mathrm{H}$ & $\times 10^{4}, \mathrm{Jm}^{-3}$ & \multicolumn{4}{|l|}{1.93} & \multirow[t]{4}{*}{ 16) } \\
\hline $\mathrm{C}_{p}$ & $\times 10^{\prime}, \mathrm{Jm}^{\cdot 3} \mathrm{~K}^{-1}$ & \multicolumn{4}{|l|}{5.74} & \\
\hline$\alpha$ & $\mathrm{Wm}^{-1} \mathrm{~K}^{-1}$ & \multicolumn{4}{|l|}{35.0} & \\
\hline$a_{11}$ & $\mathrm{~nm}$ & \multicolumn{4}{|l|}{1} & \\
\hline$D_{0}$ & $\times 10^{-4}, \mathrm{~m}^{2} \mathrm{~s}^{-1}$ & \multicolumn{4}{|l|}{1.27} & \multirow[t]{2}{*}{ 17) } \\
\hline $\mathrm{Q}$ & $\times 10^{4}, \mathrm{Jmol}^{-1}$ & \multicolumn{4}{|l|}{8.17} & \\
\hline$T_{g}$ & $\mathrm{~K}$ & 452 & 452 & 453 & 454 & 18) \\
\hline $\mathrm{U}^{b}$ & $\times 10^{4}, \mathrm{Jmol}^{-1}$ & \multicolumn{4}{|l|}{6.2} & 19) \\
\hline
\end{tabular}

written by

$$
\Delta t=\frac{\Delta x C_{\mathrm{p}} \rho \Delta T}{V \Delta H}
$$

Physical properties and other values of $\mathrm{Fe}-\mathrm{C}$ binary steel used for numerical analysis are listed in Table 3.

\section{Results and Discussions}

\subsection{Nucleation Behavior on Sample Surface}

The heterogeneous nucleation rate in classical nucleation theory ${ }^{18,20)}$ can be written as

$$
I=\frac{10^{29}}{\eta} \exp \left(-\frac{16 \pi}{3} \frac{\sigma^{3}}{k_{\mathrm{B}} T \Delta G_{\mathrm{v}}^{2}} f(\theta)\right)
$$

where $\eta$ is the viscosity of molten steel, $k_{\mathrm{B}}$ is Boltzmann constant, $T$ is temperature, $\sigma$ is the surface energy per unit area, $\Delta G_{\mathrm{v}}$ is the Gibbs free energy change for unit volume, $\theta$ is the contact angle ${ }^{19)}$ which is assumed to be depend on time and $f(\theta)$ is the contact angle factor.

The surface energy per unit area is given by ${ }^{21)}$

$$
\sigma=\alpha \frac{\Delta S_{\mathrm{f}} T}{\left(N_{\mathrm{A}} V_{\mathrm{m}}^{2}\right)^{1 / 3}}
$$

where $\Delta S_{\mathrm{f}}$ is the molar entropy of fusion, $N_{\mathrm{A}}$ is Avogadro's number, $V_{\mathrm{m}}$ is the molar volume, $\alpha$ is equal to 0.71 for bcc lattice substrate. ${ }^{18)}$ In this analysis, the primary phase was assumed to be bcc lattice structure under equilibrium phase diagram. The Gibbs free energy change of solidification $\Delta G_{\mathrm{v}}$ and the molar entropy of fusion $\Delta S_{\mathrm{f}}$ are obtained by calculation using thermodynamic database. ${ }^{15)}$

The viscosity of molten steel is given by ${ }^{22)}$

$$
\eta=10^{-3.3} \exp \left(\frac{3.34 T_{\mathrm{L}}}{T-T_{\mathrm{g}}}\right)
$$

where $T_{\mathrm{L}}$ is the liquidus temperature, $T_{\mathrm{g}}$ is the glass transition temperature in case of binary system and can be approximated by ${ }^{18)}$

$$
T_{\mathrm{g}}=0.25\left\{x_{\mathrm{L}}^{\mathrm{Fe}} T_{\mathrm{L}}^{\mathrm{Fe}}+\left(1-x_{\mathrm{L}}^{\mathrm{Fe}}\right) T_{\mathrm{L}}^{\mathrm{C}}\right\}
$$

where $x_{\mathrm{L}}^{\mathrm{Fe}}$ is the fraction of liquid of iron at nucleation temperature, $T_{\mathrm{L}}^{\mathrm{Fe}}, T_{\mathrm{L}}^{\mathrm{C}}\left(2260 \mathrm{~K}^{23)}\right)$ is the melting temperature of iron and carbon, respectively.

The nucleation temperature is defined as the temperature, on cooling, where at least one nucleation event
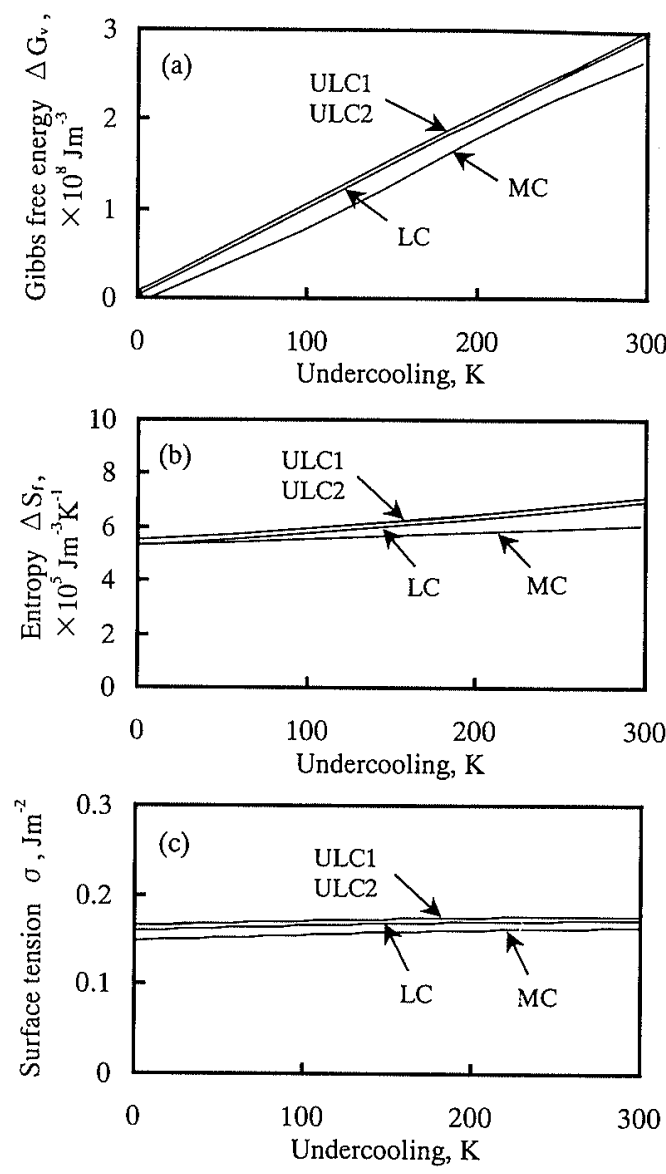

Fig. 3. Change of Gibbs free energy, entropy and surface tension with degree of undercooling.

takes place. The number of nuclei becomes in the solidification process,

$$
n=\int_{T_{\mathrm{N}}}^{T_{\mathrm{L}}} \frac{I}{\dot{T}} d T
$$

where $n$ is the number of nuclei, $I$ is the nucleation rate, $\dot{T}$ is the cooling rate, $T_{\mathrm{L}}$ is the liquidus temperature and $T_{\mathrm{N}}$ is the nucleation temperature. It is hard to determine the presence of nuclei after solidification by observation of microstructure at sample surface. As it was hard to specify the nuclei by the observation of solidification microstructure, each arm of the primary dendrite was assumed to correspond to one nuclei and the number of nuclei per unit area was determined by counting the number of primary dendrite arm per unit area. The dendrite arm spacing near the surface for sample ULC1, ULC2, LC and MC was about $1 \times 10^{-5} \mathrm{~m}$, the number of nuclei per unit area was about $1 \times 10^{10} \mathrm{~mm}^{-2}$ in this experimental range.

Figure 3 shows the relationship between the Gibbs free energy change of solidification, the molar entropy of fusion, the surface tension and the degree of undercooling from liquidus temperature for $\mathrm{Fe}-\mathrm{C}$ equilibrium binary system. The Gibbs free energy shown in Fig. 3(a) increases with increasing undercooling for all samples. The entropy of fusion shown in Fig. 3(b) increases slightly with increasing undercooling for all samples. The surface tension shown in Fig. 3(c) also increases with increasing both undercooling and carbon content. As the Gibbs free 
energy change, the entropy of fusion and the surface tension were influenced only slightly by carbon contents, it was seemed that the nucleation phenomena was little governed by carbon contents from the viewpoint of thermodynamics.

Figure 4 shows the relationship between the undercooling for nucleation and the incubation time. The incubation time for nucleation was required a finite value. The difference of incubation time for each sample was very small, and there was scarcely any effect from carbon content on incubation time in this analytical range. Therefore, it was considered that nucleation phenomena were little influenced on carbon content.

\subsection{Cooling Curves of Sample Surface during Solidifica- tion}

Figure 5 shows the measured cooling curves of sample ULC1, ULC2, LC and MC. The five measured points were chosen at intervals of $2 \mathrm{~mm}$ from the center on a line passing through the center of the round sample and the cooling curve of each point was produced from the measurements made at these points. Figure 5(a) shows measured cooling curve of sample ULC1. The start of measurement was defined as the moment which chill plate was came into contact with molten steel. The presence

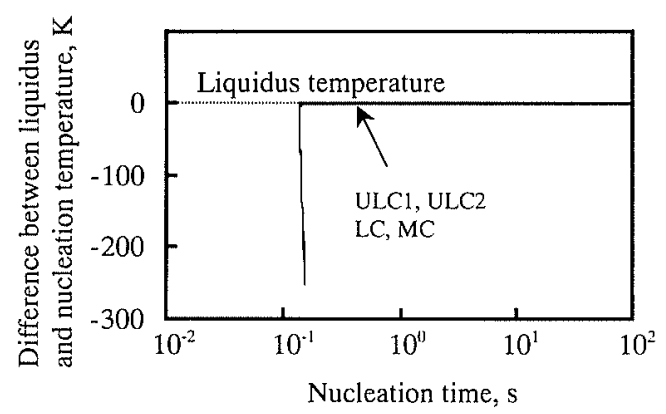

Fig. 4. Change of difference between liquidus and nucleation temperature with nucleation time.
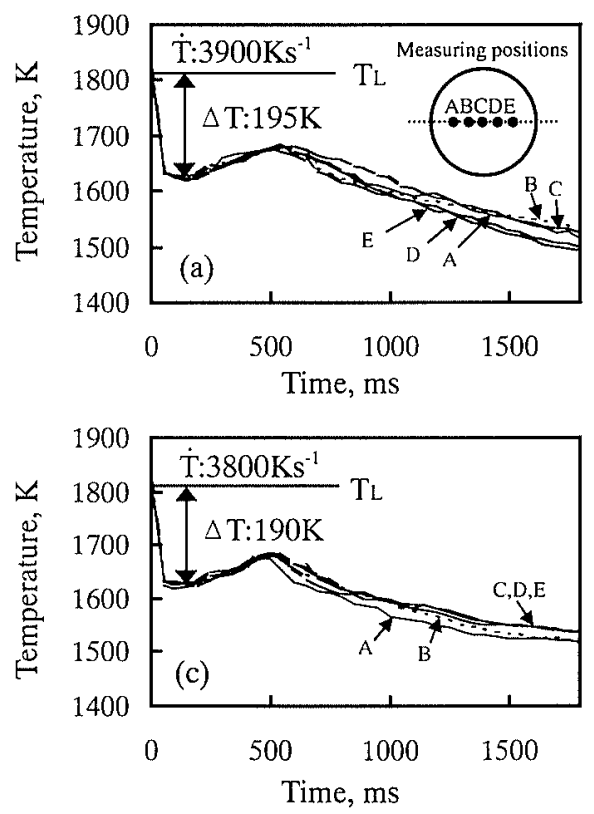

of undercooling and recalescence phenomena at the initial stage of solidification was made clear by this experiment as previous reports. ${ }^{8,24)}$ The surface temperature of each point decreased linearly with increasing time and cooling rate decreased slightly, and then temperature showed a minimum value. Then, surface temperature increased again and reached a maximum value, and decreased again. The cooling rate were defined by the slope of cooling curve at liquidus temperature, and degree of undercooling was defined by the difference between liquidus and minimum temperature on the cooling curve. The difference of temperature at each measured point was very small until the temperature reached the recalescence temperature, but the difference of temperature became larger gradually after recalescence.

Figure 5(b) shows the measured cooling curve of sample ULC2. As the cooling curve of sample ULC1, the undercooling and recalescence were observed on the cooling curve. The difference of temperature at each point was very small before recalescence, but that difference increased slightly with time after the recalescence.

The behavior of cooling curve of sample LC is shown in Fig. 5(c) as same as that of sample ULC1 and ULC2, the undercooling and recalescence phenomena were observed.

Figure 5(d) shows the cooling curve of sample MC, the behavior of the curve is the same as that of sample ULC1, ULC2 and LC. After the recalescence, the difference of measured temperature at each point was large in comparison with sample ULC1, ULC2 and LC.

The existence of both undercooling and recalescence phenomena at the initial stage of solidification were shown for all samples under the experimental conditions.

The temperature of $\delta / \gamma$ transformation could not be measured in this experiment.

Figure 6 shows the relation between the standard deviation of sample surface temperature and time. In
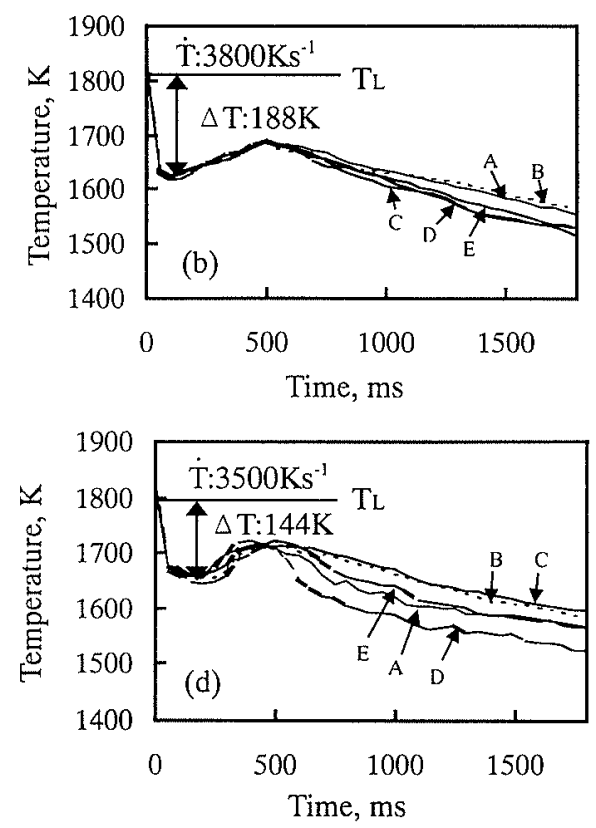

Fig. 5. Measured cooling curve.

(a) sample ULC1, (b) ULC2, (c) LC and (d) MC. 


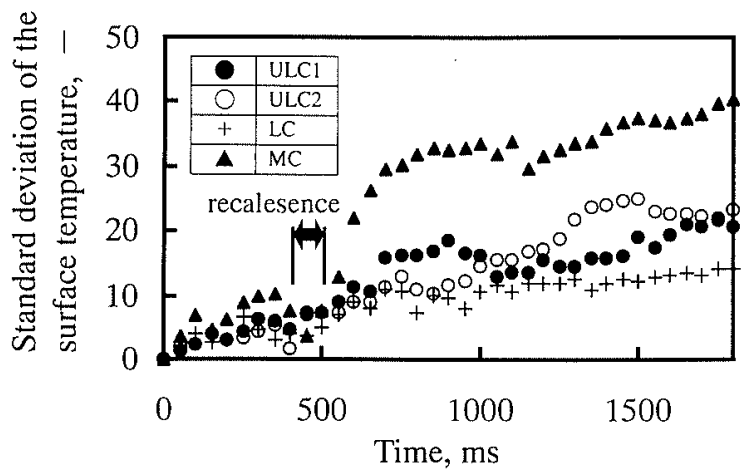

Fig. 6. Relation between standard deviation of temperature of sample surface and time.

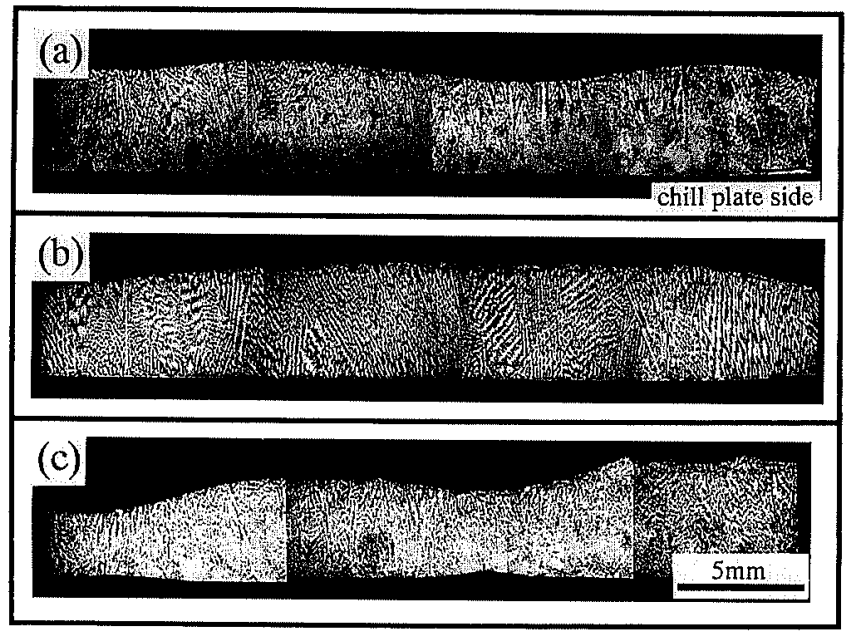

Fig. 7. Solidification microstructure.

(a) sample ULC2, (b) LC and (c) MC.

case of low value of the standard deviation, the difference of temperature at the sample surface was small and the cooling condition for sample was uniform. On the contrary, in case of large value of the standard deviation, the difference of temperature at surface was large. As any sample showed the small value of the standard deviation before about $500 \mathrm{~ms}$, the sample surface was seemed to be cooled uniformly. But that value increased gradually after about $500 \mathrm{~ms}$.

The time about $500 \mathrm{~ms}$ showed the recalescence time in Fig. 5 and the surface layer of sample was seemed to be solidified completely by this time. The standard deviation increased in this order after recalescence: sample MC, ULC and LC. The cooling conditions of sample surface before recalescence were little influenced by carbon contents, but that conditions after recalescence were depended on carbon contents.

It seemed that the nucleation phenomena were not governed strongly by carbon contents at the initial stage of solidification under the experimental conditions.

\subsection{Unevenness of Initially Solidified Shell}

The longitudinal solidification microstructure of sample ULC2, LC and MC are shown in Fig. 7. Solidification microstructure was revealed by saturated picric acid with a surface-active agent under condition that temperature was about $313 \mathrm{~K}$ and dipping time was about $180 \mathrm{~s}$, and then a photograph was taken. The mi-
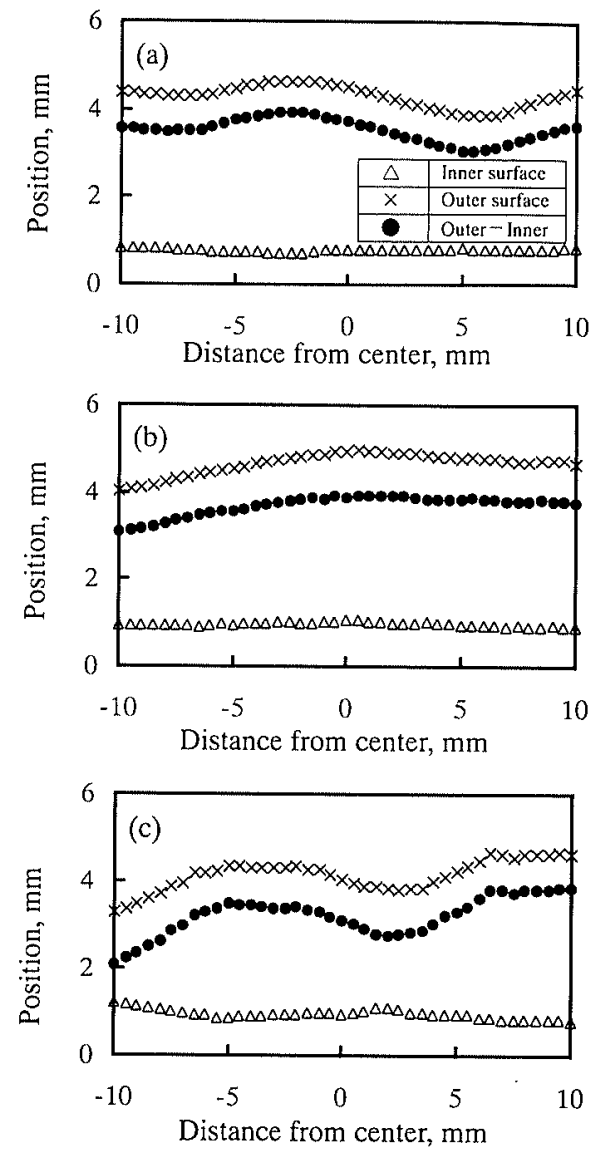

Fig. 8. Shape of solidified shell.

(a) sample ULC2, (b) LC and (c) MC.

crostructure solidified unidirectionally from chill plate side for each sample unlike the report of Kajitani et al $^{25}{ }^{25}$

Figure 8 shows the solidified shell profile of sample ULC2, LC and MC. As the outline of solidified shell was not uniform, a standard line was set at the below the shell at suitable position and the distance between the standard line and inner and outer line of the shell was measured respectively. The distance between these lines corresponded to shell thickness, which was plotted using a filled circle in Fig. 8. The shape of solidified shell depended on carbon content, the shell of middle carbon steel was non-uniform as in previous reports. ${ }^{1)}$ In order to estimate unevenness of shell thickness, next equation was used

$$
\varepsilon=\frac{\sigma}{d}
$$

where $\varepsilon$ is the degree of unevenness, $\sigma$ is the standard deviation, $d$ is the average shell thickness, respectively.

Figure 9 shows the relationship between unevenness of the solidified shell and carbon content. The degree of unevenness of sample LC was the smallest and that of sample $\mathrm{MC}$ was the biggest, and the value of sample ULC2 was between sample $\mathrm{LC}$ and $\mathrm{MC}$ in this experimental range. The degree of unevenness of sample was seemed to correspond to the deformation of initially solidified shell which occurs after the recalescence time on cooling curve, because the standard deviation after recalescence which depend on the carbon contents of sample shown in Fig. 6 increased gradually with time. 


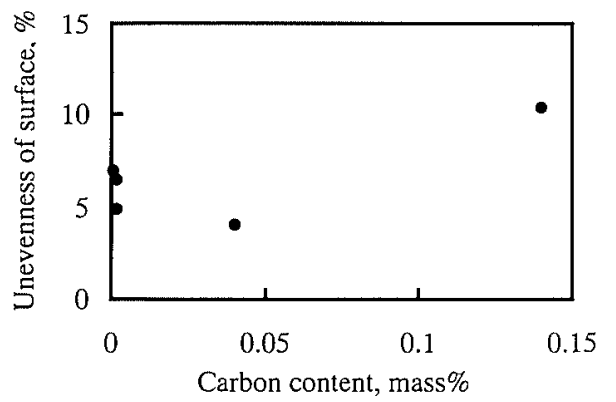

Fig. 9. Relation between unevenness of solidified shell and carbon contents.

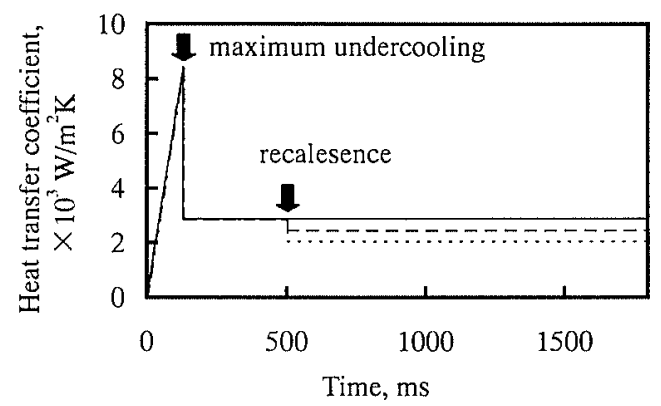

Fig. 10. Change of interfacial heat transfer coefficient used in numerical analysis for sample ULC2 with time.

\subsection{Heat Transfer between Sample and Chill Plate}

Solidification conditions near the sample surface seemed to be controlled by heat transfer between the sample and the chill plate, therefore estimation of heat transfer behavior is very important. Numerical analysis of solidification with undercooling was performed using several heat transfer coefficients. The calculated cooling curve on the sample surface was fitted to the measured cooling curve, and the heat transfer coefficient between the sample and the chill plate was estimated.

The variation of interfacial heat transfer coefficient at the initial stage of solidification was analyzed ${ }^{8,14)}$ experimentally, it was clear that the value of heat transfer coefficient increased gradually with increasing time and reached its highest value, and then decreased. The variation of interfacial heat transfer coefficient with time used in the numerical analysis for sample ULC2 is simplified and shown in Fig. 10. The value of interfacial heat transfer coefficient increases linearly with increasing time until the maximum undercooling time, and then dropped abruptly. The highest value was $8.4 \times 10^{3}$ $\mathrm{W} \mathrm{m}^{-2} \mathrm{~K}^{-1}$ and the lower value were $2.1-2.9 \times 10^{3}$ $\mathrm{W} \mathrm{m}{ }^{-2} \mathrm{~K}^{-1}$, and both of these values showed similar results. ${ }^{24,26-29)}$ The variation of interfacial heat transfer coefficient of samples ULC1, LC and MC was the same as that of sample ULC2.

Figure 11 shows the calculated cooling curve using the relation in Fig. 10. As the calculated cooling curve matched the measured cooling curve approximately, we can now predict the variation of interfacial heat transfer coefficient at initial stage of solidification of carbon steel.

Figure 12 shows the relationship between the position on solid/liquid interface and time. The solid/liquid interface moved rapidly immediately after start of solidification, because of the existence of the undercooling

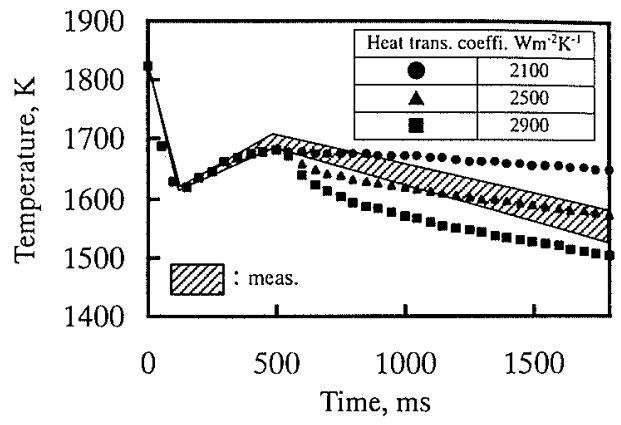

Fig. 11. Calculated cooling curve of sample surface for sample $\mathrm{ULC} 2$.

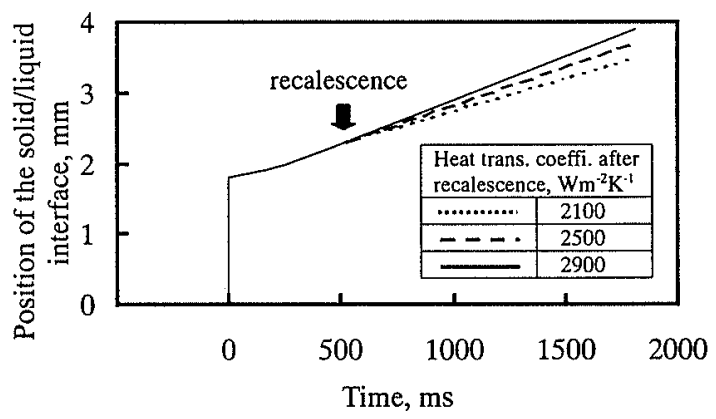

Fig. 12. Change of position of solid/liquid interface with time for sample ULC2.

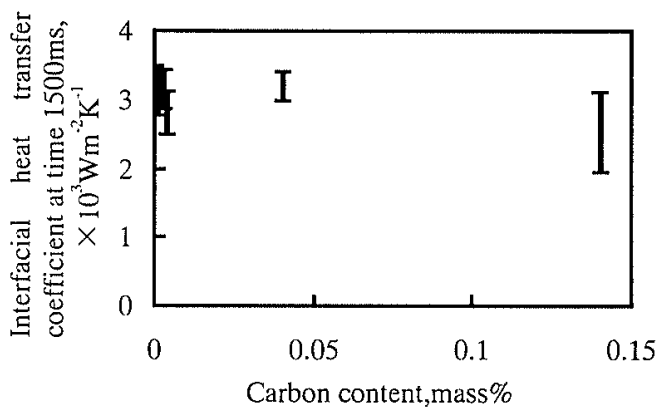

Fig. 13. Relation between interfacial heat transfer coefficient at time $1500 \mathrm{~ms}$ and carbon contents.

near sample surface. After recalescence, the position of solid/liquid interface changed with the interfacial heat transfer coefficient.

The difference of surface temperature for each measured point on cooling curve increased gradually after recalescence phenomena. In order to compare the dependence of carbon content on heat transfer coefficient, the interfacial heat transfer coefficient at time $1500 \mathrm{~ms}$ was plotted. As shown in Fig. 13, the interfacial heat transfer coefficient at time $1500 \mathrm{~ms}$ depended on carbon contents. The difference of heat transfer coefficient increased in this order : sample LC, sample ULC and sample MC. There were the same tendency of dependence of carbon content shown in Fig. 13 in both the standard deviation of temperature at time $1500 \mathrm{~ms}$ shown in Fig. 6 and unevenness of solidified shell shown in Fig. 9.

\subsection{Deformation Mechanism of Initially Solidified Shell}

Before the recalescence time, there was little dependence of the interfacial heat transfer coefficient which were calculated on the basis of measured cooling curve 
at the measuring position. On the other hand, after the recalescence time the dependence of the measuring position of the interfacial heat transfer coefficient appeared.

After the recalescence time on the cooling curve, the undercooled region near the sample surface disappeared nearly completely, and then the solidification occurred normally with the positive temperature gradient at solid/liquid interface. ${ }^{9.26)}$ The temperature of sample surface seemed to be controlled by the heat transfer to the chill plate. Therefore, the interfacial heat transfer coefficient between the sample and the chill plate seemed to be changed depending on the contact condition. When the condition of contact is good, the surface temperature of sample surface becomes lower and the interfacial heat transfer coefficient becomes higher.

The dependence of the temperature of the sample surface on the measuring position was caused by the difference of the contact condition between the sample and the chill plate. When the thermal deformation of the initially solidified layer of the sample occurs and the air gap between the sample and the chill plate is formed after the recalescence time, the contact condition becomes poor and then the temperature of the sample surface increases.

The deformation behavior of the initially solidified shell was made clear by the measurement of cooling curve on sample surface directly. The deformation of initially solidified shell of the ultra low carbon, low carbon and middle carbon steel are seemed to be caused by the thermal stress which depends on the solidification mode, that is, the ratio of $\delta$ phase to $\gamma$ phase during solidification.

\section{Conclusions}

The cooling curves for the initial stage of solidification of ultra low, low and middle carbon steel sample were measured with a new temperature measurement system, and then the deformation behavior of the initially solidified shell was made clear by the cooling curve. A numerical analysis employing the dendrite tip growth model was constructed, and fitted to the measured cooling curve, and solidification conditions were predicted.

(1) Nucleation, undercooling and recalescence phenomena existed at the initial stage of solidification of ultra low, low and middle carbon steel samples. The behavior of nucleation at the initial solidification was not dependent on carbon contents in this experimental range.

(2) The interfacial heat transfer coefficient between the sample and the chill plate changed abruptly at initial stage of solidification, because of solidification of the sample surface layer.

(3) The temperature difference of sample surface on the cooling curve appeared after recalescence. The degree of temperature difference was dependent on carbon contents, and increased in this order : low carbon, ultra low carbon and middle carbon steel sample.

(4) The evenness of solidified shell thickness was dependent on carbon content, and increased in this order: low carbon, ultra low carbon and middle carbon steel sample.

(5) The evenness of solidified shell thickness were seemed to be governed by the thermal deformation at initial stage of solidification.

\section{Acknowledgements}

The authors are grateful to Professor T. Suzuki, The University of Tokyo, for valuable comments.

\section{REFERENCES}

1) Y. Sugitani, M. Nakamura and T. Watanabe: Tetsu-to-Hgamé, 67 (1981), 631

2) M. Wolf and W. Kurz: Merall. Trans., 12B (1981), 85

3) T. Matsumiya, T. Saeki, J. Tanaka and T. Ariyoshi: Tetsulo-Hagané, 68 (1982), 1782.

4) S. Dong, E. Niyamal and K. Anzai: ISIJ Int., 35 (1995), 730.

5) H. Esaka, T. Shirakami. T. Mizoguchi and S. Ogibayashi: Tetsu-to-Hagane, 81 (1995), 631

6) W. Kurz, B. Giovanola and R. Trivedi: Acta Metall., 34 (1986), 823

7) J. Lipton, W. Kurz and R. Trivedi: Acta Metall., 35 (1987), 957.

8) H. Mizukami, T. Suzuki, T. Umeda and W. Kurz: Mater. Sci. Eng., A173 (1993), 363

9) K. Salkuma and T. Suzuki: Tetsu-to-Hagané, 81 (1995), 518.

10) H. Esaka and S. Ogibayashi: Tetsu-lo-Hagané, 84 (1998), 49

11) M. J. Aziz: J. Appl. Phys., 53 (1982), 1158.

12) R. Trivedi and W. Kurz: Acla Metall., 34 (1986), 1663.

13) W. D. Murray and F. Landis: Trans. ASME, 81 (1959), 106.

14) Z. Rivlin, J. Baram and A. Grill: Metall. Trans., 21B (1990), 1063.

15) B. Jansson, M. Schalin, M. Selleby and B. Sundman: Computer Software in Chemical and Extractive Metallurgy, ed. by C. W. Bale and G. A. Irons, The Met Soc of CIM, Quebec, (1993), 57.

16) W. Kurz and D. J. Fisher: Fundamentals of Solidification 4th. Ed., Trans. Tech. Publications, Switzerland, (1998), 293.

17) Y. Ueshima, S. Mizoguchi, T. Matsumiya and H. Kajioka: Metall. Trans., 17B (1986), 845.

18) C. V. Thompson and F. Spacpen; Acta Melall., 31 (1983), 2021.

19) H. Fujino. T. Suzuki and I. Jimbo: ISIJ In/., 37 (1997), 1107.

20) T. F. Kelly, M. Cohen and J. B. Sande: Metall. Trams., 15A (1984), 819

21) F. Saepen: Acta Motall., 23 (1975), 729

22) D. Turnbull: Contemp. Phrs.. 10 (1969), 473.

23) Handbook of Physico-chemical Properties at High Temperatures, ed. by Y. Kawai and Y. Shiraishi, ISIJ, Tokyo, (1988).

24) H. Todoroki, R. Lertarom, T. Suzuki and A. W. Cramb: Proc. ol Stcelmaking Conf.. TMS, Warrendale, PA, (1997), 667.

25) T. Kajitani, H. Esaka, M. Wakoh, H. Misumi and S. Ogibayashi: Tetsu-10-Hagane, 81 (1995), 1055.

26) H. Mizukami, T. Suzuki and T. Umeda: Tetsu-to-Hagané, 78 (1992), 767

27) W. Liu, G. X. Wang and E. F. Matthys: Int. Heat Mass Transfer, 38 (1995), 1387

28) T. Mizoguchi, S. Ogibayashi and T. Kajitani: Tetsu-to-Hagané, 81 (1995), 971 .

29) T. Suzuki, Y. Miyata, H. Sakuta and M. Ohta: Tetsu-to-Hagané, $73(1987), 289$ 